\title{
Formulas for calculating the refractive index profile of optical fibers from their transverse interference patterns
}

\author{
Kenichi lga and Yasuo Kokubun
}

\begin{abstract}
A set of successive approximation formulas for calculating the refractive index profile of optical fibers from their transverse interference patterns has been derived. The probing ray refraction due to the index gradient in the core is corrected for by these formulas, and the accuracy of index determination is improved. The error is confirmed to be less than $3 \times 10^{-3} \%$ of the index difference by means of computer simulations. The index profiles of some optical fibers are practically determined with the help of these formulas.
\end{abstract}

To widen the transmission bandwidth of multimode ${ }^{1}$ and single-mode ${ }^{2}$ optical fibers by controlling the refractive index profile, its more precise measurement is necessary. The transverse interference method has been used for determining the refractive index profile as a nondestructive method. . $^{3-8}$ This method has some merits, such as simplicity and speed of collecting data. As for the accuracy, there remained one problem, because the previous fringe-shift analysis ${ }^{9}$ neglected the effect of refraction of the probing ray due to the transverse index gradient.

In this paper, the authors have derived exact formulas which completely correct for the probing ray refraction. ${ }^{10}$ In addition, we have investigated the accuracy of these formulas and have measured the refractive index profiles of some optical fibers with an interference microscope.

It is assumed that an optical fiber is immersed in matching oil whose refractive index is equal to that of the cladding $\left(n_{2}\right)$. A test beam which consists of parallel light rays illuminates the fiber in transverse direction. The probing ray is refracted through the core which has an azimuthal symmetric refractive index profile, as shown in Fig. 1. When the $y$ axis is located on the lens-object plane, the ray incident on the fiber displaced by $y$ from the $x$ axis is focused at $y^{\prime}=y \cdot \sec \psi$

The authors are with Tokyo Institute of Technology, Research Laboratory of Precision Machinery and Electronics, 4259, Nagatsuta, Midoriku, Yokohama, 227, Japan.

Received 11 October 1977.

0003-6935/78/0615-1972\$0.50/0.

(C) 1978 Optical Society of America. in the image plane. The phase shift of the probing ray as a function of $y^{\prime}$ is obtained by observing the fringe shift in the interference pattern.

The fringe shift $R(y \cdot \sec \psi)$ in the lens-image plane was given in Ref. (11) as follows;

$$
\frac{\lambda}{D} \cdot R(y \cdot \sec \psi)=\int_{\mathrm{P}_{\mathrm{o}}}^{\mathrm{P}_{1}} n(r) d s-2 n_{2}\left(a^{2}-y^{2}\right)^{1 / 2}-n_{2} y \cdot \tan \psi,
$$

where $a$ is the core radius, $n_{2}$ is the refractive index of the cladding, $D$ is the distance between interference fringes corresponding to one wavelength, and $\lambda$ is the wavelength of the light source. The optical pathlength from $\mathrm{P}_{0}$ to $\mathrm{P}_{1}$ and the refraction angle $\psi(y)$ are expressed $^{12}$ in terms of the local refractive index $n(r)$ and the transformed argument $u=r \cdot n(r)$ by

$$
\begin{aligned}
\int_{\mathrm{P}_{0}}^{\mathrm{P}_{1}} n(r) d s=2 n_{2}\left(a^{2}-y^{2}\right)^{1 / 2}-2 \int_{n_{2} y}^{u_{2}}\left[\frac{d \ln n(u)}{d u}\right] \\
\\
\times \frac{u^{2} d u}{\left(u^{2}-n_{2}^{2} y^{2}\right)^{1 / 2}}
\end{aligned}
$$

and

$$
\psi(y)=-2 n_{2} y \int_{n_{2} y}^{u_{2}}\left[\frac{d \ln n(u)}{d u}\right] \frac{d u}{\left(u^{2}-n_{2}^{2} y^{2}\right)^{1 / 2}},
$$

respectively, where $u_{2}=n_{2} \cdot a$.

Therefore, substitution of Eqs. (2) and (3) into Eq. (1) gives an integral equation, but this is difficult to solve analytically because it includes the integral in the argument of trigonometric functions. Hunter et al. ${ }^{12}$ ignored the tan $\psi$ term in Eq. (1), but this term is of the same order of magnitude as the optical pathlength term.

In the first-order approximation, $\tan \psi$ and $\sec \psi$ in Eq. (1) are approximated by their first terms in a Taylor 


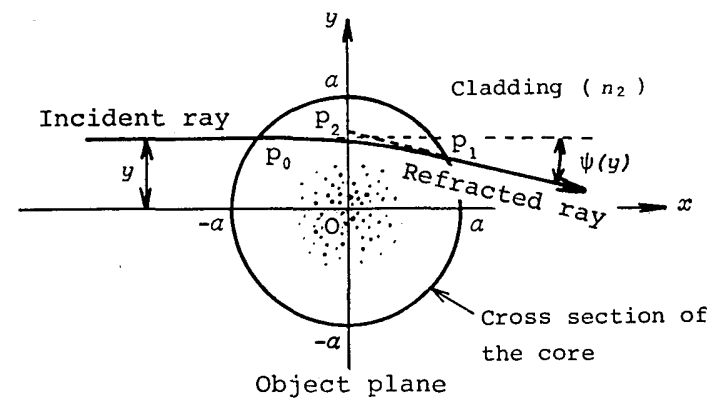

Fig. 1. Ray trajectory in a fiber which has a cylindrically symmetric index profile. [The angle $\psi(y)$ is on the order of $10^{-2} \mathrm{rad}$ in magnitude.]

series expansion. This simplification gives an Abelian integral equation, and its solution is given by

$$
n(u)=n_{2} \cdot \exp \left\{-\frac{1}{\pi} \int_{u / n_{2}}^{a}\left[\frac{\lambda}{D} \cdot \frac{d R(y)}{d y}\right] \frac{d u}{\left(n_{2}^{2} y^{2}-u^{2}\right)^{1 / 2}}\right\} .
$$

This is the formula which corrects for the ray refraction in the first order approximation.

In the second-order approximation, the refraction angle $\psi(y)$ is calculated by substituting the result of the first-order approximation into Eq. (3), and the second term in a Taylor series expansion of $\tan \psi$ is treated as a correction term. Substitution of Eq. (4) into Eq. (3) gives, after some simplification,

$$
\psi_{2}(y)=-\frac{\lambda}{n_{2} D} \cdot \frac{d R(y)}{d y} .
$$

When the corrected fringe shift in the second-order approximation is defined by

$$
F_{2}(y)=\frac{\lambda}{D} \cdot R\left\{y\left[1+\frac{\psi_{2}^{2}(y)}{2}\right]\right\}+\frac{n_{2} y}{3} \psi_{2}^{3}(y),
$$

the refractive index profile in the second-order approximation is given by

$$
n(u)=n_{2} \cdot \exp \left[-\frac{1}{\pi} \int_{u / n_{2}}^{a} \frac{d F_{2}(y)}{d y} \frac{d y}{\left(n_{2}^{2} y^{2}-u^{2}\right)^{1 / 2}}\right] .
$$

The higher order approximation can also be obtained in the same manner.

The refractive index profile can be obtained from the measured fringe shift $R(y)$ with the help of Eq. (4) or Eqs. (5)-(7).

To investigate the accuracy of Eq. (4) and Eqs. (5)(7), a parabolic index profile was initially assumed to have been given, the fringe shift $R(y)$ was numerically calculated, and the index profile was recalculated from the fringe shift with the help of Eq. (4) and Eqs. (5)-(7). The result is shown in Fig. 2.

The error $\varepsilon$ as a function of the transverse distance $r$ is defined by

$$
\varepsilon=\frac{n_{\mathrm{cal}}(r)-n(r)}{n(0)},
$$

where $n(r)$ and $n_{\text {cal }}(r)$ are the initially given profile and the calculated index profile, respectively. Since the error $\varepsilon$ of the first-order approximation is expected to be nearly proportional to $\psi^{3}$ and considering the fact that the refraction angle $\psi$ is proportional to $\Delta\{=[n(0)$ $\left.\left.-n_{2}\right] / n(0)\right\}$, it is natural to normalize $\varepsilon$ by $\Delta^{3}$. The error of $\varepsilon$ of the first-order approximation for various values of $\Delta$ is found to be nearly proportional to $\Delta^{3}$, and this expectation is proved to be right. Figure 2 illustrates the error of the previous analysis, of the first-order approximation, and of the second-order approximation, respectively. From this figure, it is found that the error of the second-order approximation is about $10^{-3}$ times smaller than that of the previous analysis.

Figure 3 shows errors for different values of the exponent $\alpha$ of the power law index profile. ${ }^{13}$ When the value of $\alpha$ is large, the error of the first-order approximation becomes rather large. However, the error of the second-order approximation is smaller than that of the first-order approximation by 2 orders of magnitude, even when $\alpha=10$.

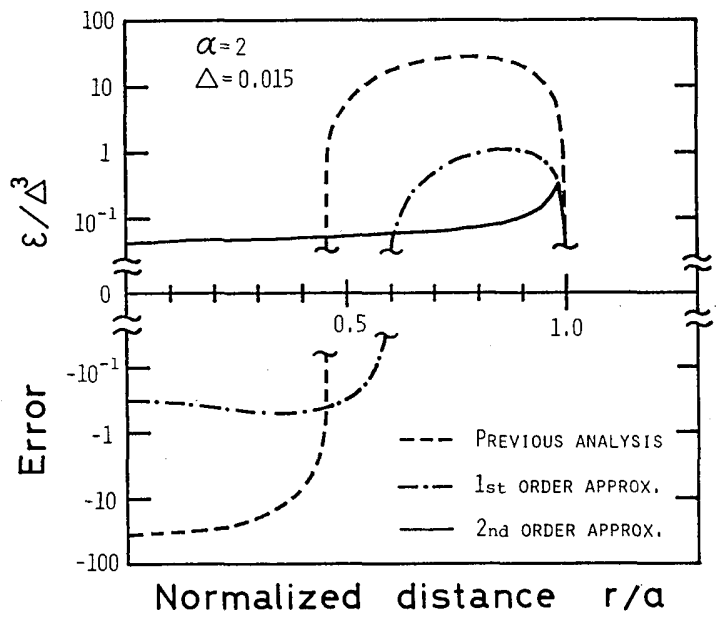

Fig. 2. Principal errors obtained by computer simulations.

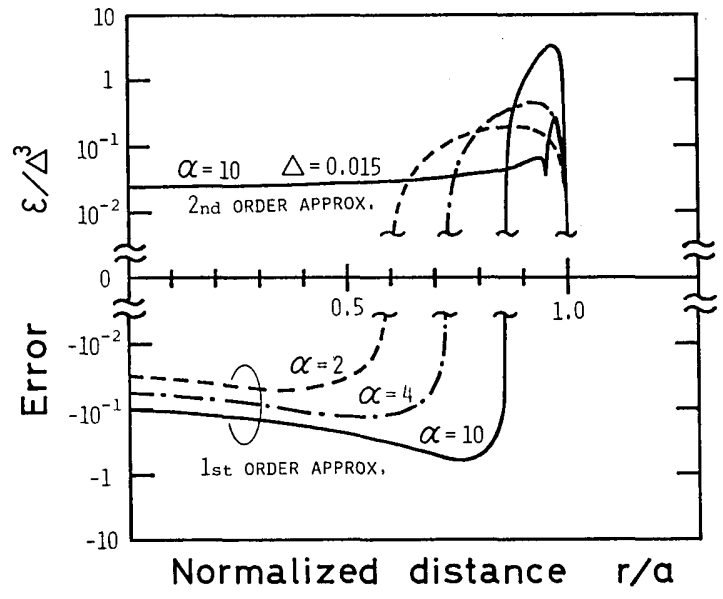

Fig. 3. Principal errors as $\alpha$ takes various values. 


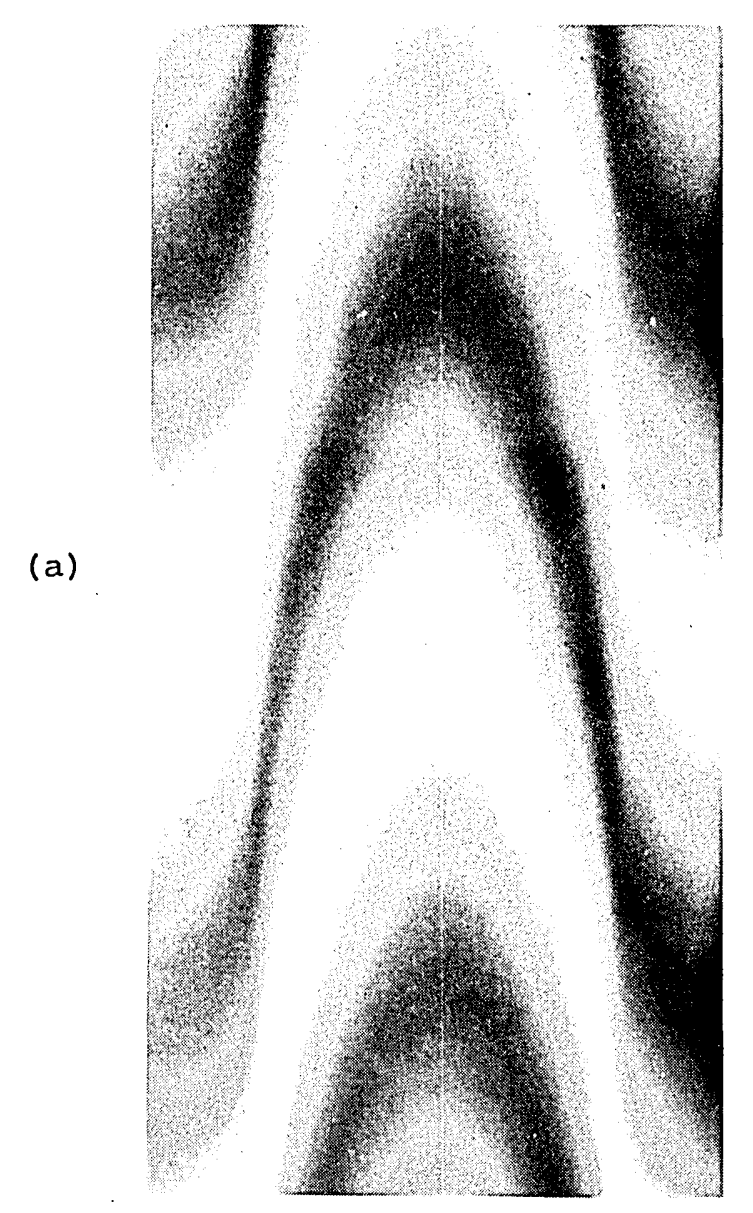

(b)

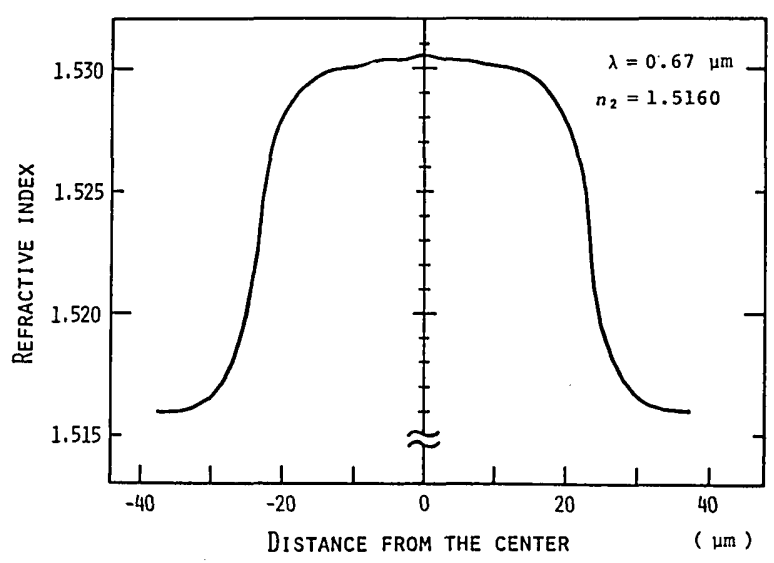

Fig. 4. (a) Transverse interference pattern of a graded index fiber made of compound glass. (b) Refractive index profile reduced from the pattern shown in (a) with the help of Eqs. (5)-(7).

From these computer simulations, the error of the second-order approximation is confirmed to be within $3 \times 10^{-3} \%$ of the index difference between the core center and the cladding.
The index profiles of some optical fibers were practically measured by using an interference microscope. The refractive index of the matching oil was made equal to that of the cladding by controlling the temperature. The transverse interference pattern of a graded index optical fiber made of compound glass is shown in Fig. 4(a). Diameters of core and cladding are $69 \mu \mathrm{m}$ and 200 $\mu \mathrm{m}$, respectively. The refractive index of the cladding was determined to be 1.5160 by comparing it with that of the matching oil. The core radius was divided into ninety-five equal parts, and the fringe shift corresponding to each divided part was used to calculate numerically the refractive index profile with the help of Eqs. (5)-(7) of the second-order approximation. The result is shown in Fig. 4(b).

In this measurement, the principle error is small. But the reading error was evaluated as $0.5 \%$ of the index difference, and this limits the total accuracy. However, some reduction of this reading error seems possible by incorporating an image processing technique, for example, the combination of a TV system and a minicomputer.

The index profile of a thin fiber whose core radius was about $4.5 \mu \mathrm{m}$ was also successfully measured, so this method seems to be applied to single-mode fibers.

In this study, a set of exact formulas for calculating the refractive index profile from its transverse interference pattern has been derived, and it was confirmed that this method can be applied to the precise measurement of index profile without destroying fiber samples. Since it is not necessary to prepare a special sample form, this method is applicable to the fast measurement of preform rods and fibers during the manufacturing process, especially for soft rods of plastic focusing fibers. ${ }^{5}$

The authors express their sincere thanks to Y. Suematsu for the valuable discussions of this work and to T. Tako for the preparation of an interferometer microscope.

\section{References}

1. R. Olshansky and D. B. Keck, Appl. Opt. 15, 483 (1976).

2. K. Jürgensen, Appl. Opt. 14, 163 (1975).

3. B. Shiraishi, G. Tanaka, S. Suzuki, and S. Kurosaki, Nat. Conv. Rec. IECE Jpn. 891 (1975).

4. K. Nishizawa, M. Toyama, and T. Fukushige, Conv. Rec. Jpn. Soc. Appl. Phys. 23a-G-1 (1975).

5. K. Iga and N. Yamamoto, Appl. Opt. 16, 1305 (1977).

6. M. E. Marhic, P. S. Ho, and M. Epstein, Appl. Phys. Lett. 26, 575 (1975).

7. M. J. Saunders and W. B. Gardner, Appl. Opt. 16, 2368 (1977).

8. F. T. Stone, Appl. Opt. 16, 2738 (1977).

9. F. D. Bennett, W. C. Carter, and V. E. Bergdolt, J. Appl. Phys. 23, 453 (1952).

10. K. Iga and Y. Kokubun, at 1977 International Conference on Integrated Optics and Optical Fiber Communication (1977), paper $\mathrm{C} 2-4$.

11. G. D. Kahl and D. C. Mylin, J. Opt. Soc. Am. 55, 364 (1965).

12. A. M. Hunter II and P. W. Schreiber, Appl. Opt. 14, 643 (1975).

13. D. Gloge and E. A. J. Marcatili, Bell Syst. Tech. J. 52, 1563 (1973). 